HUMANITAS |Sayı - Number: 4

Güz / Autumn, Tekirdağ, 2014

\title{
UNE LECTURE PSYCHANALYTIQUE DE LA PLEURANTE DES RUES DE PRAGUE: LA VOIE QUI MENE SYLVIE GERMAIN DE LA SOUFFRANCE A LA CATHARSIS
}

\author{
Emine GÜZEL ${ }^{1}$
}

\begin{abstract}
Öz: II. Dünya Savaşı sonrası kuşağında yer alan ve günümüz çağdaş yazarlarından biri olan Sylvie Germain, birçok yapıtında toplumları derinden etkileyen olayları kötülük kavramı doğrultusunda ele almıştır. Birbirinin devamı şeklinde ve aynı konular etrafında gelişen yapıtları ortak bellek olgusunu yansitmaktadır. Felsefe alanında doktora diplomasına sahip olan yazar, olayları tarihsel bağlamda dini, felsefi ve edebi boyutta derinlemesine irdelemektedir. Yazarın edebi evrenini yüzyıllardır insana gözyaşı akıttıran ve derin acılar yaşatan kötülük kavramı oluşturmaktadır. Savaş, cinayet, katliam, tecavüz, ensest, adı ne olursa olsun, kötülük sonrası yaşanan yıkımlar ve acılar yalnızca içinde bulunulan dönemi ve bireyleri etkilememiş, aynı zamanda gelecek kuşaklara da genetik yoluyla aktarılmıştır. Psikanalizin öncüsü olarak bilinen Freud, aynı zamanda bilinçaltı kavramını da ortaya koyan ilk bilim adamıdır. $\mathrm{Bu}$ buluşuyla, birçok ruhsal hastalığın temelini, bastırılmış duyguların gün yüzüne çıkmasıyla açıklamıştır. Ruhsal bir hastalık olarak ortaya çıkan psikoz, dışa aktarılamamış duygu ve düşüncelerin farazi görüntüler (halüsinasyonlar) oluşturmasıyla kendini göstermektedir. Bu hastalık bireyin iç dünyasında yaşadığı çatışmaları gerçek yaşama aktarmasıyla oluşan kopukluğu ifade etmektedir. Sylvie Germain'in Prag sokaklarında ağlayan kadın, adlı öyküsü, bir kadın anlatıcının, bilinç ve bilinçaltı savaşının ortaya koyduğu farazi bir görüntü altında insanlığın yaşadığı tüm acıları dile getirmektedir. Edebi metinlerin insanlığın yaşanmışlıklarına tanıklık eden belgeler olduğu unutulmamalıdır. Bu çalışma, ruhsal sıkıntılardan arınma yollarından biri olarak "yazma" eyleminin önemini ortaya koymaktadır.
\end{abstract}

Anahtar Sözcükler: Sylvie Germain, Edebiyat, Psikanaliz, Katharsis, Kötülük, Acı, Psikoz, Prag sokaklarında ağlayan kadın.

\footnotetext{
${ }^{1}$ Araş. Gör., Namık Kemal Üniversitesi, Fen-Edebiyat Fakültesi, Fransız Dili ve Edebiyatı Bölümü.

Doktora Öğrencisi, Ankara Üniversitesi, Sosyal Bilimler Enstitüsü, Batı Dilleri ve Edebiyatları Anabilim Dalı (Fransız Dili ve Edebiyatı Bölümü). eguzel@,nku.edu.tr
} 


\section{Introduction: Sylvie Germain et l'obsession du mal}

L'écrivain français Patrick Modiano vient de recevoir le Prix Nobel de littérature 2014. L'Académie suédoise a couronné Modiano de ce grand prix littéraire pour "l'art de la mémoire avec lequel il a évoqué les destinées humaines les plus insaisissables et dévoilé le monde de l'Occupation"'. Le secrétaire de l'Académie a ajouté que son"univers est fantastique, ses livres se répondent les uns aux autres" ${ }^{\prime \prime}$. Toutes ces remarques ont de commun avec l'univers romanesque de Sylvie Germain où la souffrance se laisse dévoiler comme la force majeure de la mémoire. C'est à travers Sylvie Germain et son récit La pleurante des rues de Prague que nous allons tenter de répondre à la question "comment soulager la souffrance" avec une approche psychanalytique.

Au cours des années 70, Sylvie Germain obtient une formation de philosophie et inspirée de la pensée d'Emmanuel Levinas, elle écrit une thèse intitulée Perspectives sur le visage: Trans-gression, Dé-création, Trans-figuration dans laquelle les thèmes du visage et de la nuit occupent une grande place, et où elle poursuit sa réflexion sur la pensée mystique déjà traitée dans son mémoire de maîtrise (Koopman-Thurlings, 2007). Il est remarquable qu'après la soutenance de sa thèse elle s'est lancée dans le monde littéraire, hantée par un désir irrésistible d'écrire: "Si je n'écris pas, je suffoque" (Goulet, 2009, p.19). Cette confession serait un point de départ pour conquérir l'univers de Germain.

Sylvie Germain est considérée comme l'une des voix les plus fortes de la littérature française contemporaine. Elle écrit depuis une trentaine d'années des romans et des essais dans une impressionnante cohérence, couronnés de nombreux prix qui dépassent largement les frontières de la France. Sylvie Germain doit sa réputation à la mise au point des problèmes qui touchent toute l'humanité. Son univers romanesque "est hantée par la grande question du mal qu'elle explore dans ses aspects les plus violents (guerres, meurtres, incestes, souffrances et douleurs les plus variées, folie), on sent qu'elle s'attache à aider ses personnages à trouver une issue aux maux qui les rongent" (Goulet, 2009, p.31).

Les auteurs de l'après-guerre, y compris Sylvie Germain, ne peuvent pas rester indifférents à la souffrance de l'homme ni à la destruction et au carnage de la guerre. L'horreur des guerres et les champs de bataille ou toute autre forme de violence et de brutalité qui déchirent les hommes, constituent le fondement historique de l'œuvre de Germain. Le talent de l'écrivaine réside dans la manière de faire entendre, par un langage très sincère, la souffrance, le chagrin et les larmes des hommes qui ne cessent depuis des siècles. Elle chante et exalte douloureusement les traces et les blessures du mal qui sont inscrites dans la

\footnotetext{
${ }^{2} \mathrm{http} / /$ www.liberation.fr/livres/2014/10/09/le-francais-patrick-modiano-prix-nobel-delitterature_1118123, consulté le 2 novembre en 2014.

${ }^{3} \mathrm{http} / / /$ www.liberation.fr/livres/2014/10/09/le-francais-patrick-modiano-prix-nobel-delitterature_1118123, consulté le 2 novembre en 2014.
} 
mémoire universelle de l'humanité et qui sont imprimées non seulement dans les écrits mais aussi dans les cœurs et les consciences. Elle s'avère donc être une chantre moderne qui veut chanter l'homme harcelé de souffrance.

La pleurante des rues de Prague est une nouvelle publiée en 1992, dans le premier cycle de l'ensemble romanesque de Germain:

Dans l'œuvre de Sylvie Germain, les romans sont véritablement premiers, nécessaires, scandant la progression de leur auteur dans sa vie, jouant leurs psychodrames sur la scène du monde un peu à la manière de fables ou de contes, et s'interrogeant sur les difficultés à être. L'ensemble peut s'articuler selon quatre périodes qui tracent un itinéraire personnel, avec, au centre du dispositif, un ouvrage singulier, qui n'est pas un roman mais un récit personnel et fantastique: La pleurante des rues de Prague, qui fait office de pivot dans cette évolution (Goulet, 2009, p. 21).

Alain Goulet, par cette affirmation, déclare qu'il y a un parallélisme entre la vie de l'auteur et ses ouvrages. La pleurante des rues de Prague en est le témoignage, puisqu'elle ancre dans la biographie de Germain à travers une narratrice homodiégétique. La narration alterne entre "je" et "elle". La ville de Prague où l'auteur a vécu pendant quelques années, la mort de son père et l'abandon de son amant se reflètent dans cette nouvelle. Ce récit apparaît comme le journal intime d'une personne psychotique qui est à la quête d'une ombre qui se montre de temps en temps par une image fantomatique. Les mots spécifiques empruntés à la psychanalyse tels que "psychotique", "ombre" et "fantomatique" décèlent un langage symptomatique qui attend d'être déchiffré. Une lecture à travers la psychanalyse mettra en évidence la compréhension de l'œuvre. Freud, qui grâce à sa méthode thérapeutique est le fondateur de la psychanalyse, ouvre une nouvelle ère par sa découverte de l'inconscient. La psychanalyse est un procédé qui donne libre cours à la parole spontanée et provoque l'émergence de l'inconscient psychique à travers une enquête libre et l'interprétation des rêves. Au début du XXe siècle, on ne parlait pas de terme de psychanalyse. Car,

(...) le psychiatre classait les symptômes et observait l'évolution, mais il n'était pas question pour lui d'"écouter" les malades. Quant à l'origine des troubles des patients, elle n'était à rechercher dans sa constitution (rôle de l'hérédité), voire dans son caractère moral. Ni la société, ni son milieu, ni sa famille ne pouvaient être tenus pour responsables (Freud, 2010, pp. 10-11).

Cependant, Freud, ne se contente pas des travaux restreints de ses collaborateurs et approfondit ses recherches. Il (2010, p.11) "découvre que les symptômes ont un sens, qu'ils sont à interpréter comme un langage que le thérapeute ne peut saisir qu'à partir de l'inconscient". C'est ainsi que Freud constate le rôle central de l'inconscient dans les phénomènes psychiques. Les désirs insatisfaits sont refoulés par le conscient dans les profondeurs sombres de l'individu où règne l'inconscient.

Il est remarquable que rien ne tombe dans l'oubli chez l'homme et que tout est enregistré et conservé dans son psychisme. Freud (2010b, p.50) affirme que 
l'oubli n'est pas "une destruction de la trace mémorielle, donc un anéantissement", et que "dans la vie psychique, rien de ce qui s'est une fois constitué ne peut sombrer, que tout demeure conservé en quelque manière et peut, dans des circonstances appropriées, par exemple du fait d'une régression allant assez loin, être appelé à resurgir" (2010b, p.51). Le manque d'oubli, loin d'empêcher l'omission des souvenirs, les conserve d'une manière étonnante pour les mettre à jour selon les circonstances. Les souvenirs, chez Sylvie Germain, surtout ceux qui sont mauvais, sont entassés dans sa caverne et ne cessent de la hanter. Elle souffre, non seulement de la mort de son père et de l'abandon de son amant qui lui causent un grand malheur, mais aussi d'un mal qui a envahi toute l'humanité sous le nom de la guerre, du crime, du viol, de l'inceste qui règnent dans le monde entier. A part ses problèmes individuels, elle est aussi tourmentée par la souffrance collective à laquelle elle ne peut rester indifférente. Elle fait couler ses larmes à l'intérieur et au bout d'un moment elle s'écrase sous "le poids du réel" (Vasse, 2008) qui la torture. C'est le moment où il y a un conflit entre le conscient et l'inconscient. L'angoisse éprouvée provoque une coupure entre la réalité et l'imagination, et dégage des images hallucinatoires. Désormais, l'inconscient prend le pouvoir et commence à se manifester. Il met au jour tout ce qui a été refoulé par le conscient, et l'état confus de la narratrice annonce, dans la nouvelle, l'arrivée d'un être fantomatique, née de ses phantasmes:

Elle s'est glissée dans le livre. Elle s'est faufilée dans les pages comme un songe s'en vient visiter un dormeur, se déploie dans son sommeil, y trame des images et mêle à son sang, à son souffle, de fin échos de voix.

Elle va partout, n'importe où, elle s'introduit où elle veut, elle traverse les murs aussi aisément que les troncs d'arbres ou que les piles des ponts. Aucune matière n'est pour elle un obstacle; ni la pierre, ni le fer, ni le bois ou l'acier n'arrêtent son élan, ne retiennent ses pas. Toute matière a pour elle la fluidité de l'eau (Germain, 1992, pp.15-16).

Cette citation montre le conflit qui règne dans le psychisme de la narratrice. L'héroïne qu'elle présente dans le prologue, est dotée de deux caractéristiques merveilleuses: matière transparente qui symbolise l'esprit invisible et forme gigantesque celui du corps visible. La matière transparente prend sa source des larmes coulées à l'intérieur et la forme gigantesque est due à la quantité énorme de sa souffrance :

Mais cette Immatérielle n'était en rien une hallucination; elle était une vision provenant de quelque mystérieuse condensation de larmes et de douleurs émanées d'hommes et de femmes, et d'enfants également, pris dans les rets du malheur (Germain, p.35).

\section{Une étude psychanalytique dans La pleurante des rues de Prague}

L'importance de la psychanalyse résulte du fait qu'elle permet à l'homme d'appréhender, à travers son histoire personnelle, les mécanismes psychiques qui se sont développés au cours de sa vie, surtout pendant son enfance, et qui caractérisent sa personnalité et conditionnent une partie de ses attitudes sans en 
être conscient. Le psychanalyste analysant la relation entre l'inconscient et le conscient a pour but d'interpréter le comportement psychique de l'homme traumatisé. L'angoisse fait d'abord du malade un névrotique qui présente des troubles psychiques. Une personne névrotique est consciente de sa souffrance et peut continuer sa vie, dans un état dépressif. Lorsque la dépression aggrave, la maladie change de dimension et prend le nom de psychose (Freud, 2010b, p.76). Freud (2010b, p.76) désigne la psychose "comme la création d'un nouveau monde en rupture avec le monde extérieur, lorsque la réalité s'est refusée au désir d'une façon grave, apparue comme intolérable". Il est remarquable qu'il y a un isolement (la catatonie) et un éloignement de la vie réelle pour créer un monde tolérable. Hallucinations, délires et langage rudimentaire sont quelques symptômes de la psychose ${ }^{4}$. Lorsqu'on analyse l'état chaotique de la narratrice qui commence à voir des hallucinations, il est clair que son inconscient a pris le pouvoir. La transition de l'inconscient au pouvoir, conduit la narratrice à la psychose. L'image d'une gigantesque femme boiteuse qui apparaît dans différents moments au "hasard" en est la preuve.

Les descendants des personnes traumatisées présentent plus de risques de développer un trouble psychologique à la suite d'une expérience anxieuse. Le malade manifeste de graves symptômes psychologiques et physiologiques sans en savoir exactement la cause. Le strabisme, le silence, le repliement sur soi apparaissent comme des symptômes physiologiques qui annoncent une défaite. Freud a observé, dans L'homme aux loups, que les traumatismes psychologiques de l'enfance laissaient des traces incontestables durant toute la vie de l'individu. Dès lors, vient à l'esprit la question de savoir, comment une mauvaise expérience d'enfance peut tellement affecter l'homme. Le problème réside dans la dénomination de la notion du "fantôme", selon le psychanalyste Didier Dumas ${ }^{5}$ :

Tant qu'un traumatisme n'est pas assumé, il est toujours vivant. Un traumatisme mental est un événement que nos structures psychiques n'arrivent pas à digérer. Un événement monstrueux, effrayant. Quelque chose que les mots ne peuvent pas rendre compte. On ne peut en parler, c'est la peur, l'effroi ou la honte! Et lorsque la honte d'y avoir été impliqué interdit d'en parler, alors on enferme cet événement traumatique dans une explication mensongère, et c'est cet événement enterré dans un mensonge qui se transmet de l'inconscient des parents à celui de l'enfant, et engendre ce que la psychanalyse contemporaine appelle un "fantôme" (Canault, 2007, p.21).

La psychanalyse joue un rôle primordial dans ces cas-là afin d'élucider la cause du problème. Le personnage gigantesque et fantomatique de la nouvelle "n'a ni nom, ni âge, ni visage" (Germain, 1992, p.19) et les rues qu'il traverse sont tout à fait sombres et sans lumière. La vision apparente fait penser à quelqu'un

\footnotetext{
${ }^{4}$ http://fr.wikipedia.org/wiki/Psychose, consulté le 2 novembre en 2014.

${ }^{5}$ Je cite le nom de Didier Dumas en me référant au livre de Nina Canault intitulé Comment payet-on les fautes de ses ancêtres.
} 
d'amnésique qui n'a plus d'identité. Que peut être son nom? Guerre, crime, meurtre, génocide, cruauté ou un mélange de tout cela. Et son âge? Puisque c'est une femme géante, elle doit appartenir à l'époque des contes, où le temps y commence par l'expression: "Il était une fois". Donc, elle vient d'un passé lointain qui a été transféré au présent par l'abolition du temps. Son visage, il n'est pas apparent non plus. Il est à préciser que le visage pour Sylvie Germain signifie la rencontre avec l'autre et fait référence à l'influence d'Emmanuel Levinas. MariskaKoopman-Thurlings (2007, p.114) définit ainsi la place du visage dans la pensée de Levinas: "Notion centrale dans l'éthique de Levinas, le visage de L'Autre est ce qui me fait sortir de moi-même, il n'est jamais donné, il reste infiniment autre". Cette constatation montre que les traits du visage peuvent déceler une nationalité et c'est la raison pour laquelle ils n'ont pas d'importance. Ce qui compte c'est l'universalité, l'humanité totale.

La pleurante des rues de Prague contient un prologue, douze apparitions et un épilogue. Cette construction formelle peut être qualifiée comme le journal intime d'un psychotique qui prend des notes pour ne pas oublier les apparitions hallucinatoires, ou comme l'agenda d'un psychiatre, qui contient des notes prises au cours d'une psychothérapie concernant son malade. Le sens de la forme ne sera éclairé qu'à la fin du livre. "Et le livre qui suit, n'étant composé que des traces de ses pas, s'en va lui aussi au hasard"(Germain, 1992, p.17).

A part l'apparence de la femme fantomatique, les choses deviennent de plus en plus confuses chez la psychotique, et une image biographique, celle du père mourant, s'entremêle à celle de la géante:

Toute la souffrance de cet homme s'engouffra dans la rue, se réverbéra dans le ciel aux éclats de métal, et mugit dans le vent. Toute la lutte muette de cet homme couché, cloué entre deux draps, sa lutte contre la mort, se révéla en cette rue déserte. Le regard de cet homme, son regard de douceur, de patience, se leva dans la rue. Tous les pavés étaient ses yeux et contemplaient l'immensité du ciel resplendissant de ténèbres bleutées. Et son visage, son visage où nulle laideur, nulle colère ni haine ni mensonge jamais n'affleurèrent, s'arracha soudain des plis de la robe de la géante (Germain, 1992, p.55).

L'état confus de la narratrice commence à se réhabiliter vers les dernières apparitions de la géante. Le retablissement ne commence à s'avérer qu'à la septieme apparition au moment où l'image da la femme géante et anéantie par des "grains de lumière". "Elle n'est cependant nullement un fantôme, une fossilisation du passé. Elle n'est pas davantage une prophétesse. Elle n'annonce rien" (Germain, 1992, p.60). Ce peu de lumière symbolise les tentatives du conscient qui veut reprendre son pouvoir. L'image de la femme, qui a surgi dans les rues, sur des collines, c'est-à-dire dans les espaces ouverts, commence à s'infiltrer dans les espaces clos d'une manière peu visible à sa huitième apparition: "Ce n'est pas seulement le long de vieilles façades de maisons à l'abandon, dans des bosquets de lilas ou à flanc de colline, qu'elle manifeste son

\footnotetext{
${ }^{6}$ L'expression est empruntée à Mikhail Bakthine dans Esthétique et théorie du roman.
} 
imprévisible présence. C'est aussi bien dans des lieux clos, - des chambres, des boutiques, des cafés" (Germain, 1992, p.65).

La manifestation "imprévisible" désigne bien l'un des symptômes de la psychose: l'hallucination. Les images hallucinatoires peuvent se manifester abruptement dans des moments et des lieux inattendus. Cette fois-ci l'image fantomatique se présente dans l'espace clos, et s'approche de plus près de la narratrice. A un moment, elles sont face à face:

Assise à la table de cette chambre d'hôte, je lisais. Une lampe en métal, (...), jetait sur le livre un ovale de lumière, et les objets posés autour du livre striaient la nappe de traits d'ombres obliques.

Je fus soudain détournée de ma lecture. Elle était là, postée au bord de la table, immobile autant qu'invisible, mais si présente qu'il était impossible de mettre en doute de sa venue. (...). Elle se tenait bien trop près pour pouvoir se montrer; plus près qu'elle ne l'avait jamais été (Germain, 1992, p.66).

La présence, qui s'introduit dans la chambre, est "invisible" et "éphémère", mais elle évoque le visage "d'une fillette aux yeux trop grands, très sombres" (Germain, 1992, p.68) sur le mur. Ce petit visage, entremêlé de celui de son père, évoque l'histoire des juifs, traumatisée et fait reference au photographe Roman Vishniac (Germain, 1992, pp.68-69). C'est la photo ci-dessous qui figure dans le récit ${ }^{7}$ :

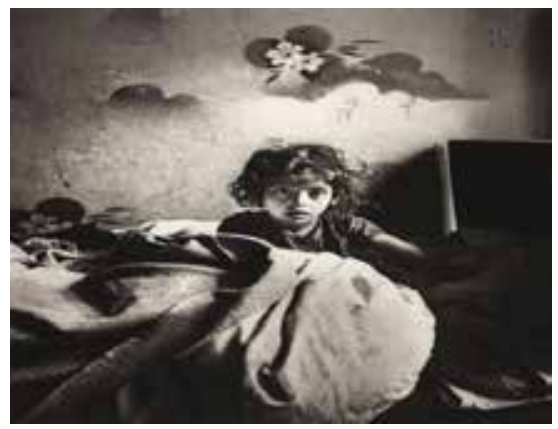

La mort de cette "fillette", suite à la misère dans une chambre sans chauffage, "fait, à jamais, une plaie de honte dans l'histoire des hommes" (Germain, 1992, p.69). La littérature comporte beaucoup d'histoires douloureuses qui montrent la cupidité et l'égoïsme de l'homme, et l'indifférence à la souffrance de l'Autre. Donc, les textes littéraires peuvent porter les marques de la honte universelle de l'homme. "Ces textes-là, qui naufragent le cœur pour mieux le rendre universel, fût-ce le temps d'un battement, la géante au pied clochant les traverse de part en part. Ses pas résonnent dans leurs mots, ses larmes luisent entre leurs lignes" (Germain, 1992, p.87).

\footnotetext{
${ }^{7}$ http://www.telerama.fr/scenes/roman-vishniac-un-artiste-revele,116957.php, consulté le 3 novembre en 2014.
} 
Sigmund Freud (1971), dans son article "La création littéraire et le rêve éveillé", montre la source des thèmes qui provoquent chez le lecteur des émotions fantasmatiques refoulées. Le philosophe entretient un parallélisme entre l'enfant qui crée un jeu et un créateur littéraire qui crée un univers imaginaire. "Le poète fait comme l'enfant qui joue; il se crée un monde imaginaire qu'il prend très au sérieux, c'est-à-dire qu'il dote de grandes quantités d'affect, tout en le distinguant nettement de la réalité" (Freud, 1971, p.5). L'enfant dans ses jeux a le désir de se faire grand en imitant "ce qu'il a pu connaître de la vie des grandes personnes. Il n'a donc aucune raison de dissimuler ce désir" (Freud, 1971, p.6). La situation change pour l'auteur dans le déploiement de son désir fantasmatique, "celui-ci, d'une part, sait qu'on attend de lui, non plus qu'il joue ou qu'il s'abandonne à sa fantaisie, mais qu'il agisse dans le monde réel; d'autre part, parmi les désirs qui sont à la base de ses fantasmes, il en est qu'il est nécessaire de dissimuler; c'est pourquoi l'adulte a honte de ses fantasmes, les sentant enfantins et interdits"(Freud, 1971, p.6). Selon Freud (1971, p.6), "les désirs non satisfaits sont les promoteurs des fantasmes" et "le fantasme vient corriger la réalité qui ne nous donne pas satisfaction".

La dixième apparition montre l'indifférence de la narratrice vers son objet fantomatique. Elle refuse de regarder dans ses yeux pour ne pas en être captée. Elle veut se délivrer de cette image fantasmagorique qui la hante "depuis des siècles". Cet emploi hyperbolique du temps (depuis des siècles) fait référence à l'archétype du mal et à la mémoire.

Ses yeux presque transparents, au regard suppliant, dévorant la lumière, consumé de pitié. Des yeux que l'on ne peut croiser sans perdre la raison,- et qu'imaginer déjà, suffit à navrer la pensée. Des yeux dont le regard vous saisit à la gorge et ne vous lâche plus, vous enlace avec tant de douceur que l'on garde jamais l'envie de pleurer, de pleurer de ses larmes à elle (Germain, 1992, p.87).

La mémoire décomposée par le conflit de l'inconscient et du conscient commence à se recomposer. Le regard introspectif de la psychotique, tourné vers les sombres profondeurs de l'imagination, s'y détache pour rejoindre la raison, c'est-à-dire le regard prospectif. La malade qui est en état de guérison, sort de son milieu clos pour rentrer dans la vie actuelle. Elle cesse de pleurer pour ne pas fournir de matière à ses fantasmes. La psychotique veut enfin détruire son image hallucinatoire pour s'en délivrer complètement.

Fut-ce l'odeur de son corps d'Immatérielle qu'exhala la terre du chantier, (...). Ce ne pouvait être qu'elle, même si je ne la vis pas. Un instant sa présence se fit palpable dans l'air. Elle venait de passer, légère, rapide, portée par le vent du soir (Germain, 1992, pp.96-97).

L'apparition "imprévue" de la géante qui se manifestait fréquemment jusqu'à la dernière apparition a mis une césure d'une année avant de disparaître. La douzième apparition représente la dernière. "Et puis un jour, soudain, par une claire matinée d'octobre, elle a reparu. Mais elle n'a reparu que pour mieux disparaître. Ce fut un adieu muet, et très bref" (Germain, 1992, p.105). 
Le récit a commencé par la première apparition de la géante "un soir d'automne", il a continué dans la linéarité du temps réel suivant les autres apparitions et s'est terminé avec la douzième apparition dans la "claire matinée d'octobre". L'obscurité et l'ombre font allusion à l'inconscient, la clarté et la lumière au conscient. Se basant sur ce constat, il est possible de dire que le récit commence par l'avènement de l'inconscient, continue par son règne et se termine par sa chute. Lorsqu'on pense au niveau du conscient, au début, il est détrôné de son royaume par l'inconscient et expulsé vers le lointain, ensuite il tâche de se rétablir durant le temps et la fin annonce sa victoire par la reconquête de son royaume. Le triomphe du conscient sur l'inconscient représente la guérison de la psychose.

La dernière partie, "épilogue", annonce le rétablissement total d'une maladie mentale qui n'évoque aucune image apparente. La disparition de l'image de la géante nourrie d'une grande souffrance universelle et des larmes coulant depuis des siècles, et l'arrêt des pleurs signifient la catharsis de la malade.

Elle a quitté la ville. Les saisons passent, la pluie, la brume, le soleil, la neige rythment les jours de Prague, mais elle demeure absente.

Sa haute silhouette ne surgit plus nulle part, ni dans les ruelles de la VieilleVille, ni dans les faubourgs, ni sur les berges où s'attroupent les cygnes et les canards, ni à flanc de la colline de Vyšehrad ou de Petř́n (Germain, 1992, p.113).

La catharsis vient du grec katharsis qui veut dire "purgation, purification". La Poétique d'Aristote (1990, p.93) traite de la catharsis en l'attribuant à la finalité de la tragédie qui "par l'entremise de la pitié et de la crainte, accomplit la purgation des émotions de ce genre". Selon la psychiatrie, la catharsis c'est la décharge des affects qui troublent le sujet. Le psychiatre en employant la méthode cathartique "recherche la décharge des affects pathogènes et cherche à faire revivre au sujet les traumatismes" ${ }^{8}$. Cette méthode donne au malade l'occasion de se confronter au sujet refoulé qui le hante constamment. La narratrice de La pleurante des rues de Prague atteint à la catharsis après avoir décrit l'image fantasmagorique qui la hantait depuis longtemps. La disparition de cette image à la fin du livre prouve le décharge de la narratrice: "Elle est sortie du livre qu'elle laisse inachevé, en fiche" (Germain, 1992, p.127).

\section{Conclusion}

Sylvie Germain est avant tout une écrivaine qui ne peut rester indifférente à la souffrance de l'Autre. En se référant à Freud, il ne serait pas incorrect d'interpréter de psychotique l'écrivaine qui souffre d'un malaise où règne le mal. Son épanchement sur l'injustice des meurtres et sur les dégâts de la guerre se reflète d'une manière récurrente dans ses ouvres. La situation chaotique du monde réel ne trouve aucune solution ni explication ni par la philosophie, ni par

\footnotetext{
${ }^{8}$ http://www.geopsy.com/cours_psycho/lexique_de_psychanalyse.pdf, consulté le 3 novembre en 2014.
} 
le raisonnement dans le monde romanesque de Sylvie Germain. Le soulagement affleure dans la réconciliation entre le monde intérieur (l'inconscient) du personnage et son monde extérieur (le conscient).

On constate qu'il y a maintes façons de parvenir à la catharsis; à savoir en regardant un spectacle au théâtre, en écoutant une belle chanson, en causant avec un psychiatre ou en écrivant ses angoisses. C'est cette dernière méthode qu'emploie Sylvie Germain. Les notes qu'elle prenait à la manière d'un journal intime composent cette nouvelle fantasmagorique. Elle avait déjà affirmé dès ses débuts son moyen d'arriver à la catharsis: "écrire".

\section{BIBLIOGRAPHIE}

Aristote (1990). Poétique, introduction, traduction nouvelle et annotation de Michel Magnien. Paris: Classique de poche.

Bakthine, M. (1978). Esthétique et théorie du roman. (Traduit du russe par Daria Olivier). Paris: Gallimard.

Canault, N. (2007). Comment paye-t-on les fautes de ses ancêtres, L'inconscient transgénérationnel. Paris: Desclée De Brouwer.

Freud S. (2010). L'homme aux loups. Paris: Editions Payot\& Rivages.

-------- (2010b). Le malaise dans la civilisation. (Traduit de l'allemand par Bernard Lortholary). Editions Points.

(1971). "La création littéraire et le rêve éveillé".Essais de psychanalyse appliquée. (Traduit de l'allemand par Marie Bonaparte et Mme E. Marty). Consulté le 23 octobre en 2014, http://classiques.uqac.ca/classiques/freud_sigmund/essais_psychanalyse_appliq uee/04_creation_litteraire/creation_litteraire.pdf.

Germain, S. (1992). La pleurante des rues de Prague. Paris: Editions Gallimard.

Goulet, A. (2009). L'œuvre de Sylvie Germain. Cengiz Ertem (Ed.). Littera, XXIV, (Numéro Spécial: Sylvie Germain). Ankara: ÜrünYayınları.

Koopman-Thurlings, M. (2007). Sylvie Germain la hantise du mal. Paris: L'Harmattan.

Le Nobel de littérature attribué à Patrick Modiano (9 octobre 2014). Le Soir. Consulté le 2 novembre en 2014, http://www.lesoir.be/675685/article/culture/livres/2014-10-09/nobel-litteratureattribue-patrick-modiano.

Psychose. Consulté le 2 novembre en 2014, http://fr.wikipedia.org/wiki/Psychose.

Vasse D. (2008). Le poids du réel, la souffrance. Paris: Editions du Seuil.

Vocabulaire de psychanalyse. Consulté le 3 novembre en 2014, http://www.geopsy.com/cours_psycho/lexique_de_psychanalyse.pdf. 
Yasmine, Y. (25 septembre 2014)."Roman Vishniac, un artiste révélé". Art \& Scènes, Télérama. Consulté le 3 novembre en 2014, http://www.telerama.fr/scenes/roman-vishniac-un-artiste-revele,116957.php.

\title{
PSYCHOANALYTICAL READING IN THE WEEPING WOMEN ON THE STREETS OF PRAGUE: THE WAY THAT LEADS SYLVIE GERMAIN FROM THE SUFFERING TO THE CATHARSIS
}

\begin{abstract}
Sylvie Germain, one of the writers of the generation of the post-World War II years, is a contemporary writer who highlights in her novels the events that profoundly influenced nations through the notion of the evil. Her novels follow one another on the same subject and reflect around a collective memory. The author who holds a doctorate in philosophy, probes historical event deeply from their religious, philosophic and literary angles. Her literary universe is made of the concept of evil that has determined the humanity to cry and suffer for centuries. War, crime, murder, rape, incest - each represents the evil. The sufferings and the destructions lived after the evil had only some fatal consequence at that time, but also their effects were genetically transferred to future generations. Freud, who is considered as the pioneer of psychoanalysis, was the first scientist who elaborated the theory of the unconscious. He was then able to explain the causes many mental disorders as manifestations of repressed feelings. Psychosis, considered as a mental disorder, is the unearthing of hallucinatory images stemming from repressed feelings and thoughts. The disorder reveals a disconnect between the internal world and the real life of the individual. The short story of Sylvie Germain, entitled The weeping women on the streets of Prague, presents all the sufferings of the humanity by a narrator who describes the conflict between the unconscious and the conscious through hallucination. We should not forget that the literary texts are documents witnessing the real-life experiences. The present study attempts to show that one of the ways for catharsis is to "write".
\end{abstract}

Keywords: Sylvie Germain, Literature, Psychoanalysis, Catharsis, Evil, Suffering, Psychosis, The weeping women on the streets of Prague. 